\title{
Numerical simulation of two-component flow fluid - fluid in the microchannel T- type
}

\author{
A.A. Shebeleva ${ }^{1, a}$, A.V. Minakov ${ }^{1,2}$, A.A. Yagodnitsina ${ }^{2}$ and V.G. Andyuseva ${ }^{1}$ \\ 1 Siberian Federal University, Krasnoyarsk, Russia \\ 2 Kutateladze Institute of Thermophysics, Novosibirsk, Russian Federation
}

\begin{abstract}
Results of testing methodology for calculating two-phase flows based on the method of fluid in the cells (VOF method), and the procedure for CSF accounting of surface tension forces in the microchannel are considered in the work. Mathematical modeling of two-component flow fluid -fluid in the T- microchannel conducted using this methodology. The following flow regimes studied slug flow, rivulet flow, parallel flow, dispersed (droplet) flow, plug flow. Comparison of numerical results with experimental data done. Satisfactory agreement between the calculated values with the experimental data obtained.
\end{abstract}

\section{Introduction}

Multiphase microflows play an important role in many natural, biological and technical systems. Their exact quantification is necessary for a variety of practical applications, such as production, transportation and processing of hydrocarbons. The behavior of liquids in microsystems can not be considered as fully explored, it can differ significantly from the macroscopic currents, since it is characterized by the other ratio of viscous, surface and inertial forces [1]. The two-phase flow regime in the micro and minichannel is found in a large number of modern technological and industrial devices using gas-liquid flow. In the case of twophase flow in microchannels capillary force significantly influence the velocity of the gas bubbles. Such a flow regime as an object of study is very complex, especially in the non-circular cross-section channels with sharp corners. Calculation of two-phase flows and macroscopic channels is quite a challenge [2]. The complexity of the micro-channels description is strongly increasing, due to the essential role of interaction of the fluid with the wall. In this regard, the development of efficient and reliable numerical simulation of two-phase flows methods in microchannels is an extremely urgent task.

Literature review has shown that there are many different approaches to the modeling of fluid flows with moving boundaries. The most complete review of methods for solving problems with moving contact edges can be found in [3-7].

All numerical algorithms of solution of the movable boundary according to the type of the grid can be divided into three large groups: Lagrange, Euler and meshless methods. The meshless method does not use the computational grid, but only surface mesh, or data collection for the calculation and analysis of results are used.

The Lagrangian algorithms computation nodes and cells move together with a continuous medium. This approach enables the most precise description of the contact boundary and accurately accounts complex boundary conditions on it, for example, it takes into account the effect of surface tension. However, the use of the Lagrangian approach requires grid recalculation at each time step, which can be very expensive. Furthermore, the use of Lagrangian methods can lead to a significant distortion of computational cells, which causes significant errors in the calculation, because the shape of the movable boundary and the trajectory of its movement is often very complex.

Euler methods are promising as they use fixed, often orthogonal and uniform computational grid for calculations. Tracking the interface in this group of methods is carried out by means of continuous or discrete Lagrangian Euler markers. According to the method of tracking the contact boundary, Euler methods can be divided into three large groups: point tracking methods, interface tracking methods and interface capturing methods. Currently the most widely used are interface tracking methods.

The Volume-of-Fluid method in the cells (VOF) was is the most popular to date, thanks to its efficiency and ease of implementation, including algorithms of continuous interface capturing methods [8].

This method is considered the most effective method of solving problems with the free surface today. The purpose of this paper is to test the numerical methods and to study two-component fluid-fluid flows in the T- microchannel.

${ }^{1}$ Corresponding author: an_riv@ mail.ru 


\section{CFD MODEL}

As mentioned above, the numerical methods are used in this paper to simulate two-phase flows in a T- microchannel, based on the volume of fluid method in the cells [8]. This works well for the calculation of macroscopic flows with free surface [9-10]. The idea of the method is that the liquid and gas are treated as a single two-component medium and the spatial distribution of the phases within the computational domain is determined using a marker function $F(x, y, z, t$, , , which value specifies the volume fraction of the liquid phase in the computational cell as follows:

$$
F(x, y, z)=\left\{\begin{array}{l}
0, \text { if the cell is empty } \\
1, \text { if the cell is completely filled with the liquid }
\end{array}\right.
$$

$0<F(x, y, z, t)<1-$ if the phase boundary passes through the cell.

Since the free surface moves together with the liquid, tracking of the free boundary in space is performed by solving the transport equation for the volume fraction of the liquid phase in the cell:

$$
\frac{\partial F}{\partial t}+V \cdot \nabla F=0
$$

Here $\boldsymbol{V}$ is the velocity vector of the two-phase medium obtained by solving the hydrodynamic equations, consisting of the equations of conservation of mass, or the continuity equation:

$$
\frac{\partial \rho}{\partial t}+\nabla(\rho \boldsymbol{V})=0
$$

and the equations of motion and the law of conservation of momentum:

$$
\frac{\partial \rho V}{\partial t}+\nabla(\rho V \times V)=-\nabla p+\nabla(T)+F
$$

Where $\tau$ - viscous stress tensor, $\boldsymbol{F}$ - vector of body forces, $\mathrm{p}$ - static pressure, $\rho$ - the density of the two-phase medium. The components of the viscous stress tensor $\tau_{i j}$ defined as:

$$
\tau_{i j}=\mu\left(\frac{\partial U_{i}}{\partial x_{j}}+\frac{\partial U_{j}}{\partial x_{i}}-\frac{2}{3} \delta_{i j} \frac{\partial U_{k}}{\partial x_{k}}\right)
$$

Where $\mu$ - viscosity coefficient of the liquid, $U_{i j}$ - the components of the velocity vector.

The density and viscosity coefficient of the two-component medium considered obtained from the volume fraction of liquid in the cell:

$$
\begin{aligned}
& \rho=\rho_{1} F+(1-F) \mu_{2} \\
& \mu=\mu_{1} F+(1-F) \mu_{2}
\end{aligned}
$$

Here, $\rho 1, \mu 1$ - density and viscosity coefficient of the liquid, $\rho 2, \mu 2$ - the density and viscosity coefficient of the second fluid.

Thus obtained density values $\rho$ and viscosity $\mu$ are included in the equation of motion and determine the physical properties of the two-phase medium. The phenomenon of surface tension occurs when considering the fluid flows with the interface, which cannot be ignored in the case of flow in microchannels. Surface tension plays a key role in microchannels. The study of currents, controlled by surface tension, is a very difficult task. VOF method makes it relatively easy to take into account the effect of surface tension forces.Most often, the VOF method uses the so-called continuum surface force (CSF) algorithm to model surface tension [11] The essence of this algorithm is that the equations of motion include an additional volume force $F_{s}$ whose magnitude is given by the relation:

$$
F_{S}=\sigma k \nabla F
$$

Where $\sigma$ is the surface tension coefficient and $k$ is the curvature of the free surface determined by the divergence of the normal vector:

$$
k=\nabla\left(\frac{n}{|n|}\right)
$$


The normal to the free surface is in turn calculated as the gradient of the volume fraction of the liquid phase in the cell:

$$
n=\nabla F
$$

On the solid wall, the magnitude of the normal vector is determined from the contact angle $\theta$ :

$$
n=n_{w} \cos \theta+\tau_{w} \sin \theta
$$

Where $n_{w}, \tau_{w}$ - are the normal and tangential vectors to the wall.

The methodology used is described in [9.12]. In this paper, we highlight the numerical methods. The difference analog of convection-diffusion equations can be obtained by means of finite volume method for the multi-block structured grids, the application of which automatically conservatism of the resulting circuit. Counter flow scheme of second order QUICK is used for the convective terms approximation of hydrodynamic equations (3). Implicit scheme of the first order approximation is used for time-dependent terms of hydrodynamic equations. The diffusion flux and source terms are approximated by the second order of accuracy. The relationship between the velocity and pressure fields is implemented using SIMPLEC procedures at combined grids. This approach allows overcoming the difficulties described above with movable interfacial boundary resolution. The resulting system of differential equations is solved iteratively using multigrid solver.

\section{Mathematical formulation}

The flow of immiscible liquids in the T-microchannel was considered. The geometry of the channel is shown in Figure 1.

The dimensions of the microchannel: $a=1000$ micrometers, $b=200$ micrometers, $c=2000$ micrometers, d=400 micrometers, f=200 micrometers. After the upper channel supplied with water. Through second channel - kerosene. The fluid inputs are shown in Table 1. The physical properties of liquid are density, viscosity, respectively: kerosene: $780 \mathrm{~kg} / \mathrm{m} 3,0.0024 \mathrm{~kg} / \mathrm{m} * \mathrm{~s}$; water: $998.2 \mathrm{~kg} / \mathrm{m} 3,0.001003 \mathrm{~kg} / \mathrm{m}^{*} \mathrm{~s}$. The surface tension on the surface of the water-kerosene was set to $0.0487 \mathrm{~N} / \mathrm{m}$.

The structured computational grid consisting of 500000 was used for calculation. The following inlet boundary conditions were set: mass flow input (Table 1), Neumann condition output (derived from the normal value of zero) in the output.

Figure 1. The geometry of the channel

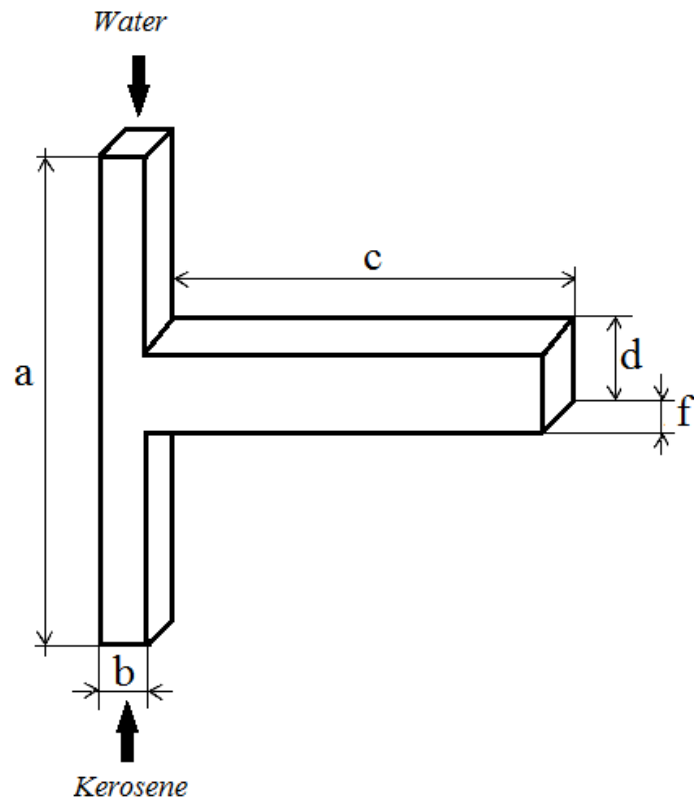

Table 1. The value of expenses

\begin{tabular}{|l|c|c|}
\hline flow regime & $\begin{array}{c}\text { Water flow rate, } \\
\text { microliters per minutes }\end{array}$ & $\begin{array}{c}\text { Kerosene flow rate, } \\
\text { microliters per minutes }\end{array}$ \\
\hline Slug flow & 1300 & 24 \\
\hline Plug flow & 4000 & 4000 \\
\hline Parallel flow & 960 & 240 \\
\hline Dispersed (droplet) flow & 10 & 2400 \\
\hline Rivulet flow & 4000 & 240 \\
\hline
\end{tabular}

Initially, calculations were made for the flow of pure water in the channel concerned, to verify the method of calculation. We considered two values of Reynolds number $\mathrm{Re}=30$ and 120. Experimental data were obtained using a micro-PIV method. A detailed study of the flow of water is described in the works of [13-15]. 
Qualitative comparison of theory and experiment in the central section of the channel for $\operatorname{Re}=30$ and 120 are shown in Figure 2 .
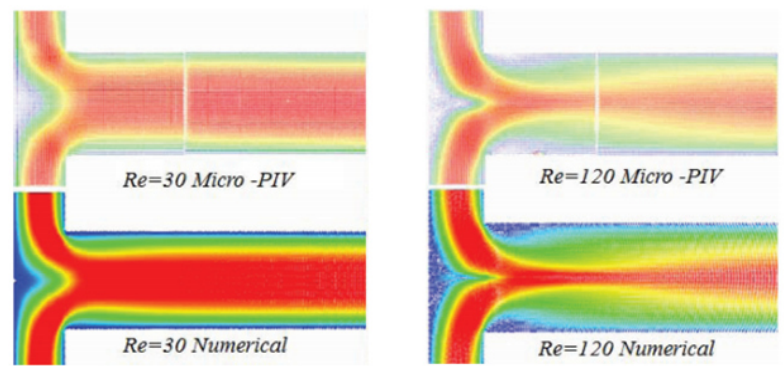

Figure 2. Average experimental and calculated velocity field in the central section of micromixer for Reynolds numbers equals 30 and 120

A quantitative comparison is shown in Figure 3. As it can be seen from the graph, agreement between the experimental and calculated data is quite good the maximum error does not exceed $10 \%$ [13].

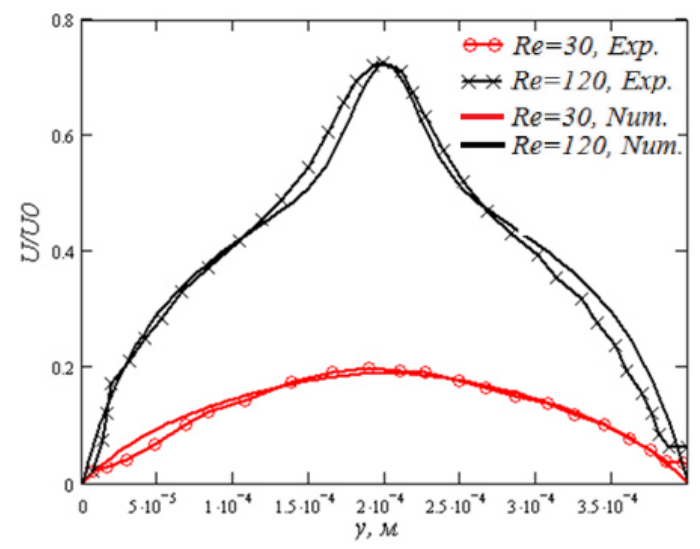

Figure 3. Velocity profiles in the central cross section of the channel

\section{Results and discussion}

The flow pattern is shown in Figure 4-8, the relationship of rate and wetting contact angle equal to $90^{\circ}$ are presented in Table 1. The calculated results were compared with experimental data obtained at the Institute of Thermophysics S.S. Kutateladze, using micro-PIV method.

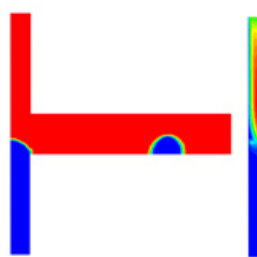

a)

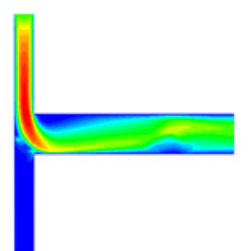

b)

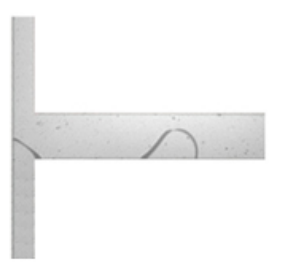

c)

Figure 4. Slug flow: a) density; b) speed module; c) experiment.

A slug flow regime is observed for water flow of 1300 microliters per minute and kerosene flow rate of 24 microliters per minute (Figure 4). This mode is characterized by the formation of individual drops of kerosene, which move along the channel wall. Results of the comparison of calculation with experimental photographs flow in the microchannel is shown in Figure 4. As it can be seen, there is a satisfactory agreement between the calculated values with the experimental data.

A slight deviation of the drop is due to the fact that at the moment the value of the contact angle is determined in the experiment with the error, and this affects the accuracy of the calculation. It is difficult to achieve the perfect match, because the process of calculating the transient and time recorded in the experimental photography is hard to "catch".

Rivulet flow mode was observed with increasing water flow of 4000 microliters per minute and kerosene flow of 240 microliters per minute (Figure 5). In this mode the kerosene flow moves in a jet form along the channel walls. This liquid stream is not continuous, but is accompanied by tears. As it is seen in Figure 5, there is a satisfactory agreement between the calculated values with the experimental data. 


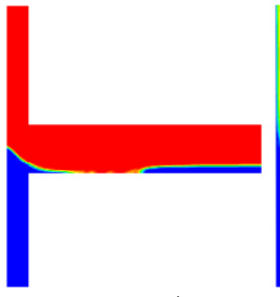

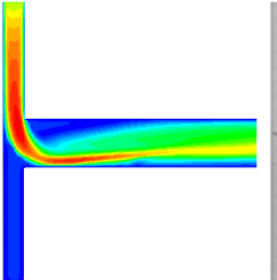

b)

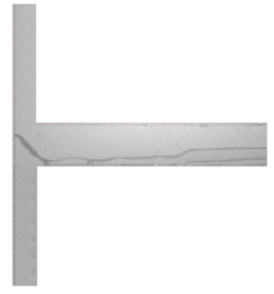

c)

Figure 5. Rivulet flow: a) density; b) speed module; c) experiment.

Parallel flow regime is observed for the water flow of 960 microliters per minute and kerosene flow rate of 240 microliters per minute (Figure 6). Kerosene flow rate is higher in parallel mode and kerosene flows continuously along the wall. At the same time, the flow is unsteady and is accompanied by oscillations of the free surface. This fact is confirmed by experimental data.

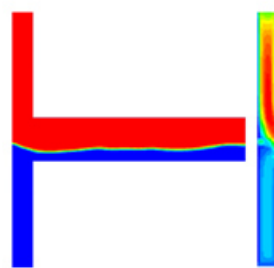

a)

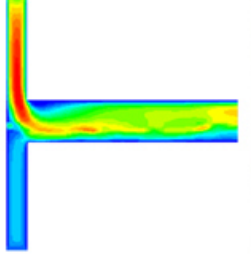

b)

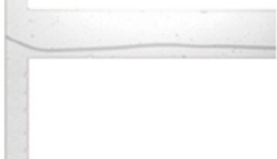

c)

Figure 6. Parallel flow: a) density; b) speed module; c) experiment.

Droplet flow regime (Figure 7) for the water flow of 10 microliters per minute and kerosene flow of 2400 microliters per minute was observed. Water consumption in comparison with kerosene is very small in the dispersed (droplet) mode. This flow regime is characterized by the formation of individual droplets that fly off from the wall and move into the stream periodically.

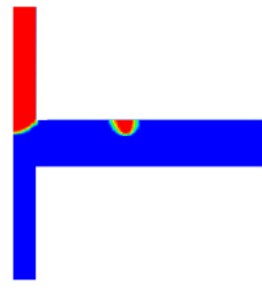

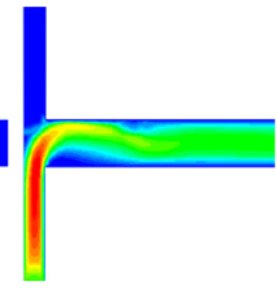

b)

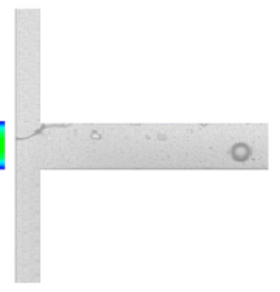

c)

Figure 7. Dispersed (droplet) flow: a) density; b) speed module; c) experiment.

Very interesting flow regime was obtained with the same value of water flow of 4000 microliters per minute and kerosene flow of 4000 microliters per minute at the inputs (Figure 8). The interface is not flat but is a serpentine surface. Interestingly, this mode is stationary. Formation flow is due to the development of Kelvin-Helmholtz instability in Plug mode. A similar phenomenon was observed at a Reynolds number greater than 145, in the study of the water flow in the microchannel [15].

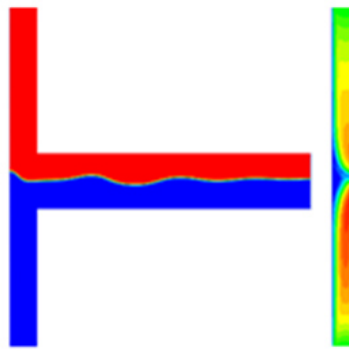

a)

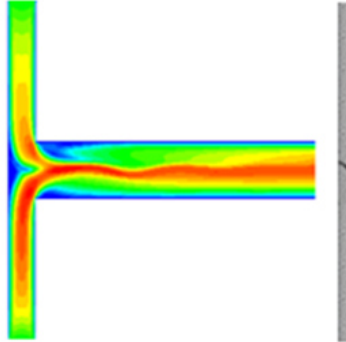

b)

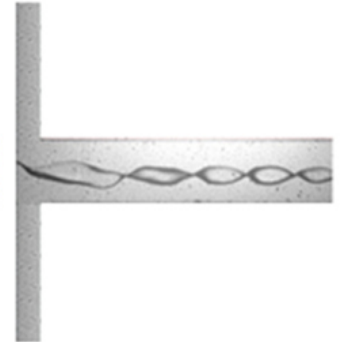

c)

Figure 8. Plug flow: a) density; b) speed module; c) experiment.

\section{Conclusion}

The investigation of two-component liquid-liquid flows in the confirmed was conducted. The structure of flows for several modes with different ratios of water and kerosene rates at the entrance to the microchannel. We received the following flow conditions: slug flow, rivulet flow, parallel flow, dispersed (droplet) flow and plug flow. Generally, on the test results, it was shown that the numerical method describes well the experiment.

\section{Acknowledgements} 00961 A).

This work was supported by Russian Ministry of Education (project № 3100) and Foundation for Basic Research (14-08- 


\section{References}

1. V. Rudyak, A. Minakov. Micromachines. Volume 5, Issue 4, 886-912, (2014).

2. D. V. Guzei, A. V. Minakov, M. I. Pryazhnikov, A. A. Dekterev. Thermophysics and Aeromechanics, January 2015, Volume 22, Issue 1, pp 61-71

3. N.G. Burago. The thesis for the degree of Doctor of Science, 222, (2003).

4. O.M. Belotserkovsky, Y.M. Davydov. M. Science. 391, (1982).

5. G.S. Khakimzyanov, Y.I. Shokin V.B. Barakhnin, N.Y. Shokina. Novosibirsk. Publishing house SP RAS. 393, (2001).

6. D.B. Kothe, W.J. Rider. S.J. AIAA. Paper - 96, (1996).

7. S. Osher, J.A. Sethian. Journal of computational physics. Vol. 79. 12, (1988).

8. C.W. Hirt, B.D. Nichols. Journal of computational physics. № 39. 201-226, (1981).

9. A.V. Minakov. Computational Mathematics and Mathematical Physics. Volume 54, Issue 10, 1560-1570, (2014).

10. A.V. Minakov. Siberian Journal of Industrial Mathematics. № 4 (36). 95-105, (2008).

11. J.U. Brackbill, D.B. Kothe, C.A. Zemach. J. Comput. Phys. 100, 335- 54, (1992).

12. A.A. Gavrilov, A.V. Minakov, A.A. Dekterev, V.Y. Rudyak. Journal of Applied and Industrial Mathematics 559-568, (2011)

13. A.V. Minakov, V.Y. Rudyak, A.A. Gavrilov, A.A. Dekterev. Thermophys. Aeromech. 19, 385-395, (2012).

14. A.V. Minakov, V. Rudyak, A. Dekterev, A. Gavrilov. J. Heat Fluid Flow 43, 161-169, (2013).

15. A. Minakov, A. Yagodnitsina, A. Lobasov, V. Rudyak, A. Bilsky. La Houille Blanche 5, 12-21, (2013). 